Mayra Chávez-Courtois

Chelsea Graham ${ }^{2}$

Irma Romero-Pérez ${ }^{1}$

Georgina Sánchez-Miranda ${ }^{1}$

Bernarda Sánchez-Jiménez ${ }^{1}$

Otilia Perichart-Perera ${ }^{1}$

\section{Experiencia y percepciones de la diabetes gestacional y su automanejo en un grupo de mujeres multíparas con sobrepeso}

Experiences, perceptions and self-management of gestational diabetes in a group of overweight multiparous women
Abstract Gestational diabetes mellitus (GDM) is a public health problem in Mexico and diet therapy is the main form of treatment. Self-management abilities are required to control the disease. Five women with GDM were studied to assess GDM risk perception and experiences related with self-management practices. Sociodemographic data were obtained and in-depth interviews were conducted and subsequently analyzed using Atlas ti V.5 software. The results revealed that women were conscious regarding the role of diet and physical activity in improving GDM control, and about the perinatal risks associated with the disease. Adherence to diet recommendations was partial, but gradual and positive lifestyle changes were observed. Emotionally, perception about having GDM was a key factor with respect to adhering to the diet. In conclusion, the medical and dietary treatment influences the cultural food behavior of women with GDM. Health professionals should consider sociocultural determinants when designing and implementing treatment strategies.

Key words Gestational diabetes mellitus, Food behavior, Sociocultural determinants, Perception, Self-management
Resumen La diabetes mellitus gestacional (DMG es un problema de salud pública en México, cuya primer línea de tratamiento es la dietoterapia. Se requieren habilidades de automanejo para el control de la enfermedad. En este trabajo se estudiaron cinco mujeres con DMG, quienes manifestaron su percepción de riesgo de la presencia de la DMG y vivencias en algunas prácticas del autocuidado. Se obtuvieron datos sociodemográficos; se realizaron entrevistas en profundidad, las cuales se analizaron con el paquete Atlas ti V.5. Los resultados demuestran que las mujeres están conscientes del papel que juega la alimentación y la actividad física en mejorar el control de su enfermedad, además de los riesgos perinatales a los que se asocia la DMG. El cumplimiento de las recomendaciones dietéticas en su mayoría fue parcial, pero se demuestran cambios positivos y graduales en el estilo de vida. Las percepciones desde las emociones que les produce la DMG, fue factor clave para que llevaran a cabo la dieta. En conclusión, el tratamiento médico y dietético influye en el comportamiento cultural alimentario de las mujeres con DMG. Los profesionales de la salud deben tomar en cuenta los determinantes socioculturales al diseñar e implementar estrategias de tratamiento.

Palabras clave Diabetes mellitus gestacional, Conducta alimentaria, Determinantes socioculturales, Percepción, Automanejo 


\section{Introducción}

La diabetes mellitus gestacional (DMG), definida como la intolerancia a los hidratos de carbono reconocidos por primera vez durante el embarazo $^{1}$, se ha venido estudiando desde hace décadas sobre todo en el campo médico y de la nutrición. La DMG aumenta las complicaciones de la madre como del producto. En la madre, la enfermedad aumenta el riesgo a desarrollar preeclampsia y la frecuencia de cesáreas. En el caso del producto, la DMG se asocia con macrosomia (peso al nacer $>4000 \mathrm{~g}$ ), distocia de hombros/ trauma obstétrico, mortalidad perinatal, hipoglucemia al nacer y obesidad infantil en etapas posteriores de la vida ${ }^{2}$. La presencia de la DMG también puede traer consecuencias a largo plazo en la madre, siendo que el 50\% de ellas tienen un mayor riesgo a desarrollar Diabetes Mellitus Tipo 2 (DM2) en los siguientes cinco años, y tienen una mayor probabilidad de desarrollar DMG en un siguiente embarazo ${ }^{3,4}$.

Se sabe que uno de los principales factores de riesgo asociados a la DM y a la DMG, son el sobrepeso y la obesidad ${ }^{5,6}$. En ese sentido la población mexicana tiene un alto riesgo a desarrollar ambos tipos de diabetes. En México, en el año 2000 la tasa nacional de DM fue del $7 \%$ en adultos en general y $7.3 \%$ en mujeres, siendo una de las principales causas de muerte. Asimismo, en el 2006, el 50\% de las mujeres entre 20 y 29 años de edad y el $70 \%$ de 30 a 39 años tenían sobrepeso y obesidad ${ }^{6}$.

El aumento en las cifras de obesidad es consecuencia de cambios en el estilo de vida que se han dado durante la última década,cambios negativos en el patrón de alimentación,aumentando el consumo de alimentos con alto contenido de energía, colesterol, grasas saturadas, azúcares y sodio, entre otros nutrimentos ${ }^{7}$. Dentro de la literatura los argumentos biomédicos son los que sobresalen al tratar de explicar los factores determinantes de la DM. Existen algunos reportes que incluyen factores de tipos ambientales, socioculturales, y de herencia, como determinantes de la enfermedad. En mujeres chilenas se han descrito como factores de riesgo el llevar una vida sedentaria, un patrón de alimentación occidental, tener sobrepeso y obesidad en la etapa pregestacional, el factor económico, así como la edad y la herencia (que un pariente de primer grado tenga diabetes) $)^{8}$.

Jones y colaboradores realizaron una revisión de estudios que exploró las creencias de salud y las conductas del estilo de vida de mujeres con
DMG. Se encontró que las mujeres que habían presentado DMG anteriormente, percibían un riesgo mayor a desarrollar DM2 a futuro, traduciéndose en conductas alimentarias y de actividad física más adecuadas en comparación con aquellas que no tuvieron el antecedente 9

En otros trabajos se han considerado algunos saberes de mujeres con DMG, respecto al significado de la patología y etiología de su enfermedad. Dichos saberes permiten a la mujer comprender su padecer y le dan sentido, lo cual permea en el tipo de cuidado que llevan a cabo ${ }^{10,11}$. En tres estudios realizados en mujeres con DMG, mexicanas ${ }^{12,13} \mathrm{y}$ americanas ${ }^{14}$, las participantes mencionaron como causas de su enfermedad al estrés, el susto, el embarazo mismo, la alimentación, causas genéticas y falta de ejercicio ${ }^{12-14}$. En mujeres hispanas de Estados Unidos, con antecedentes de DMG, se observó que el cumplimiento a las recomendaciones de alimentación fue menor en comparación con aquellas sin antecedentes de la enfermedad ${ }^{15}$. La falta de adherencia a las recomendaciones de alimentación parece estar relacionada con el contexto cultural, económico y con factores psicológicos. Mujeres con DM2 han reportado que la falta de apoyo familiar, el sentimiento de soledad, y priorizar las necesidades de otros miembros de la familia antes que las de ellas, son algunos motivos que les impide adherirse adecuadamente a su tratamiento ${ }^{16}$. A pesar de que la actividad física es otra de las recomendaciones en el tratamiento de las mujeres con $\mathrm{DMG}^{17,18}$, en ocasiones no es del todo practicada, paralelo al incumplimiento de las indicaciones dietéticas y médicas ${ }^{15}$. Dentro de las dificultades que describen las mujeres con DMG para la realización de ejercicio se incluyen: la fatiga, el dolor, la vergüenza sobre su apariencia, la falta de conocimiento sobre la capacidad de hacer ejercicio durante el embarazo, la falta de lugares accesibles para realizar la actividad, el mal clima, la inseguridad o el puerperio ${ }^{14}$. Es importante mencionar que existe información en mujeres mexicanas con DMG que describa las razones de llevar a cabo o no las recomendaciones de alimentación y actividad física, y la mayor parte de la literatura mencionada describe las prácticas de riesgo y de alimentación desde un enfoque generalizado.

En ese sentido, y siendo la DMG un problema de salud pública importante, estamos obligados a seguir comprendiendo desde el contexto sociocultural de las mujeres con DMG el intento en realizar los cambios para lograr el automanejo que se requiere en el tratamiento de la DMG. De lo cual surge la necesidad de visualizar la prác- 


\section{Material y métodos}

Este trabajo se realizó en el Instituto Nacional de Perinatología (INPer), el cual es un Instituto federal de atención de tercer nivel que se ubica en la Ciudad de México. Entre sus áreas de atención se encuentran los embarazos de alto riesgo. La DM representa el tercer diagnóstico más frecuente de la consulta obstétrica subsecuente $(5.2 \%$ de la población que acude $)^{19}$. Entre los factores de riesgo de DMG se encuentran la edad (> 25 años), tener obesidad, el número de gestas y la herencia familiar (antecedentes de DMG en otro embarazo o de DM en algún familiar ${ }^{20}$.

Es de aclararse que el análisis de los resultados cualitativos que se presentan en este trabajo se derivan del proyecto "Autocuidado en diabetes gestacional: Asociación con factores clínicos y socioeconómicos". La muestra total del proyecto fue de 30 mujeres gestantes, la cual fue seleccionada por conveniencia, y que incluyó a mujeres con DMG que fueron atendidas por el Departamento de Dietética entre noviembre del 2008 y febrero del 2009. Los criterios de inclusión fueron: tener aproximadamente entre 30-34 semanas de gestación (SDG), llevar por lo menos tres semanas siguiendo un plan de alimentación proporcionado por el servicio de dietética, y ser mayor de 18 años de edad. Se excluyeron mujeres gestantes con diagnóstico de DM tipo 1 y 2 y aquellas que como parte de su tratamiento requirieron el uso de insulina. A estas mujeres se les aplicó un cuestionario de salud adaptado de una escala utilizada y validada por el Michigan Diabetes Research and Training Center. Dicho instrumento evalúa los factores demográficos, sociales, comorbilidades, actitudes y creencias, dieta, asesoría en nutrición, auto-reporte y dificultades para el autocuidado de la diabetes, entre otros. La información en cuanto al diagnóstico, antecedentes gineco-obstétricos y de riesgo, se obtuvo a partir del expediente médico.

Del total del grupo de mujeres, se seleccionaron cinco mujeres de acuerdo a su experiencia de salud-enfermedad por manifestar: a) mayor per-

cepción de riesgo y preocupación de la presencia de la DMG; b) interés en el control de la misma; c) antecedentes de la enfermedad (dos mujeres presentaron diabetes gestacional y tres antecedentes familiares de la enfermedad).

Para obtener la información cualitativa se aplicó la metodología fenomenológica ${ }^{21}$ con el fin de conocer las percepciones de mujeres gestantes respecto a su conducta de autocuidado: alimentación y actividad física. Para lo cual se realizaron y audiograbaron entrevistas en profundidad con un guión cuyo contenido se basó en los resultados del cuestionario de salud previamente aplicado. Es pertinente mencionar que las mujeres entrevistadas acudieron al Instituto de salud, debido a su diagnóstico de DMG, por lo cual al ingresar se les dio tratamiento médico y dietético.

Las entrevistas fueron realizadas por expertos (antropólogos y psicólogos sociales), la duración aproximada de cada entrevista fue de una hora y media. Se realizó observación y notas de campo, las cuales se utilizaron para completar la información de las entrevistas. Para el análisis, primero se codificó el contenido de las narrativas por temas, y posteriormente el análisis de estas últimas se realizó con el apoyo del paquete Atlas ti V.5, el cual es un software que permite organizar la información con base a categorías de análisis previamente creadas considerando cada una de las temáticas del guión de entrevista. Los temas que se analizaron para el presente trabajo fueron: 1) Percepciones de la diabetes: definición y causas; 2) Percepciones de la presencia del diagnóstico; 3) Percepción de los Riesgos por la presencia de la DMG; y 4) Conductas de autocuidado: cambios en la alimentación y en la actividad física

Dentro de los aspectos éticos, a cada mujer se le entregó carta de consentimiento informado y se utilizaron seudónimos para proteger la privacidad de las informantes. Asimismo se aclara que el trabajo fue aprobado por los Comités de Investigación y Ética del INPer.

\section{Resultados}

La mayoría de las informantes eran casadas, sólo una era soltera; tres de ellas se dedicaban al hogar y dos recibían remuneración por trabajo. Respecto a su historia reproductiva todas por lo menos habían cursado dos embarazos; pero sólo dos de ellas refirieron haber presentado óbitos o abortos (ver Cuadro 1). En relación al diagnóstico durante el embarazo actual, cuatro de ellas presen- 
taron sobrepeso, y sólo una mujer presentó obesidad antes del embarazo. El tratamiento de todas las mujeres se basaba en la dietoterapia, y fueron atendidas en el servicio de endocrinología.

\section{Percepciones de la diabetes: definición y causas}

Por ser mujeres que se atienden en el Instituto de salud y quienes llevan un tratamiento especializado en el mismo, todas ellas tienen cierta idea de lo que es la DMG en términos médicos. En su mayoría definieron su enfermedad como altos niveles de azúcar en la sangre que sólo se presenta durante el embarazo, aunque temían que persistiera después de ese periodo reproductivo:

Ahi en las pláticas nos dijeron que es cuando uno tiene mmm alto nivel de azúcar en la sangre, que eso es lo que es la diabetes (Clara, $35 \mathrm{SDG}$ )

Además mencionaron que la enfermedad no es grave, siempre y cuando se controle con dieta, lo que nos hace referencia que están conscientes que la DMG podría causar mayor daño cuando no se siguen las recomendaciones adecuadas.

[...] una etapa nada más del embarazo y que es mala... pero si se controla uno no hay problema, mientras tu lleves tu dieta como debe de ser no hay problema. (Silvia, 30 SDG)

En este grupo de mujeres, tres fueron los factores de riesgo de DMG que nombraron: 1) el tipo de la alimentación, 2) antecedentes de la enfermedad, y 3) el sobrepeso. Respecto a la alimentación, mencionaron que la comida con alta cantidad de grasa como las hamburguesas, papas, comida de la calle y con alta cantidad de azúcar como los refrescos y dulces son detonadores de la DMG, además de la manera en cómo comían -rápido y sin horario fijo-. Una de las informantes expresó la causa de diabetes de la siguiente manera:

Yo siento que es como por comer alimentos con mucha azúcar, o porque una no tiene determinadas horas, por ejemplo antes yo mal pasaba mucho,yo no desayunaba casi por lo regular. Y comía, pero muy rápido, más bien llegaba yo a cenar; malos hábitos alimenticios y desorden de horarios. (Clara, 35 SDG)

Algunas comentaron que aunado a los malos hábitos de alimentación también la herencia de esta enfermedad (ya sea antecedentes propios o de familiares) puede ser origen de la DMG. Una de las mujeres dijo:

Cuadro 1. Características de las mujeres embarazadas con diabetes mellitus gestacional (DMG) $(n=5)$.

\begin{tabular}{|c|c|c|c|c|c|c|c|}
\hline Caso & $\begin{array}{l}\text { Edad } \\
(\text { años })\end{array}$ & $\begin{array}{c}\text { Estado } \\
\text { civil }\end{array}$ & Escolaridad & Ocupación & $\begin{array}{c}\text { Gestas } \\
(\mathrm{G})\end{array}$ & $\begin{array}{c}\text { Antecedentes de } \\
\text { riesgo }\end{array}$ & $\begin{array}{c}\text { Diagnóstico } \\
\text { durante el } \\
\text { embarazo actual }\end{array}$ \\
\hline Raquel & 32 & Casada & Secundaria & Ama de casa & $\begin{array}{c}\text { G: } 3 \text {, } \\
\text { Óbitos: } \\
2\end{array}$ & $\begin{array}{c}\text { Familiares con } \\
\text { diabetes }\end{array}$ & $\begin{array}{c}\text { Obesidad antes del } \\
\text { embarazo }\end{array}$ \\
\hline Clara & 39 & Casada & Preparatoria & Ama de casa & G: 3 & $\begin{array}{c}\text { Antecedentes de } \\
\text { intolerancia a la } \\
\text { glucosa }\end{array}$ & $\begin{array}{c}\text { Sobrepeso al inicio } \\
\text { del embarazo }\end{array}$ \\
\hline Marta & 33 & Casada & Preparatoria & Secretaria & G: 2 & $\begin{array}{c}\text { Antecedentes de } \\
\text { diabetes gestacional } \\
\text { e hipertiroideismo }\end{array}$ & $\begin{array}{c}\text { Sobrepeso al inicio } \\
\text { del embarazo }\end{array}$ \\
\hline Eleonora & 27 & $\begin{array}{l}\text { Madre } \\
\text { Soltera }\end{array}$ & Secundaria & $\begin{array}{c}\text { Dependiente } \\
\text { en tienda de } \\
\text { abarrotes }\end{array}$ & G: 2 & $\begin{array}{c}\text { Familiares con } \\
\text { diabetes y } \\
\text { antecedentes de } \\
\text { diabetes gestacional }\end{array}$ & $\begin{array}{c}\text { Sobrepeso al inicio } \\
\text { del embarazo }\end{array}$ \\
\hline Silvia & 27 & Casada & Secundaria & Ama de casa & $\begin{array}{c}\text { G: } 2 \text {, } \\
\text { Abortos: } \\
1\end{array}$ & $\begin{array}{c}\text { Familiares con } \\
\text { diabetes }\end{array}$ & $\begin{array}{c}\text { Sobrepeso al inicio } \\
\text { del embarazo }\end{array}$ \\
\hline
\end{tabular}

Fuente: Expedientes clínicos proporcionados por el Servicio de Archivo Clínico y las fichas de identificación de las mujeres embarazadas. 
Pues para mí, es este la alimentación y hereditario, yo siento, yo creo que sí, entonces, por la alimentación yo tengo la culpa porque yo me dejé mucho, como cosas que a lo mejor no, bueno en exceso (risa nerviosa), y por cuestión hereditaria ¡ni modo!, me tocó una mamá diabética, no la puedo cambiar. (Eleonora, $32 \mathrm{SDG}$ )

Otra mujer, con antecedentes de dos óbitos incluye al sobrepeso en la lista de causales del padecimiento:

Pues, en mis palabras, si es por herencia, por sobrepeso, sobre todo por malos hábitos alimenticios ¿no? el sobrepeso, es de, pues también tiene que ver por la herencia o sea hereditaria, o sea que uno viene de gente que tiene esta enfermedad, o sea en mis palabras es eso, la diabetes. (Raquel, 39 SDG)

Todas ellas se sentían culpables por padecer DMG, sobre todo por el tipo de conductas de alimentación que han llevado durante su vida antes del embarazo -. Sólo una mujer con antecedente de DMG no sabía aún las causas de la presencia del padecimiento. Ninguna de las mujeres mencionó la falta de actividad física como factor determinante de la enfermedad.

\section{Percepciones de la presencia del diagnóstico}

En su mayoría las mujeres al saberse embarazadas esperaban tener un proceso reproductivo sin complicaciones, a pesar de que algunas tenían una historia pasada de DMG. No obstante, experimentaron por lo menos un sentimiento de preocupación, tristeza, desesperación, confusión, depresión o miedo al enterarse del diagnóstico, siendo común la expresión del ¿por qué yo?. Clara colocó el miedo como el sentimiento central:

Pues al principio sí estaba un poco triste cuando me dijeron, así como que tenía miedo de comer. La verdad sí, siempre tenía miedo ya no sabía ni qué comer, llega un momento en que dije estará bien esto, estará mal, como que sí me preocupé. Como que sí al principio era así de que no, no, no o sea nada o sea ni siquiera verlo así casi, ya no todo lo que tenía azúcar (35 SDG)

Los sentimientos de preocupación no sólo estaban relacionados con el consumo de aquellos alimentos con altas cantidades de azúcar, sino también se detectó ansiedad por las posibles consecuencias que pudiera causarle al hijo que esperaban:

¡Ay! pues síme sentí mal si me sentí mal porque dije: '‘ay, por qué yo!' 'por qué yo' o sea, sí se siente uno mal más que nada por el bebé, porque yo tenía esa idea de que al bebé, estando en mi vientre le iba a pasar algo, porque he escuchado casos de que salen que muy grandotes y que no sé qué, y uno se pone a pensar no pues, que la burla de la gente, luego cómo es la gente, cómo se van a burlar de mi bebé, van a decir: 'ay, este bebé' o sea, como que te vienen muchas ideas a la cabeza (Silvia, 30 SDG)

Dos de la informantes con antecedentes de presencia de DMG en su penúltimo embarazo, pensaron que al presentar nuevamente la enfermedad (en el último embarazo) serían vulnerables a presentar DM tipo 2. Una de ella, Eleonor, estaba consciente del riesgo de volver a presentar DMG, además que en este último embarazo lo inició con sobrepeso:

Ah! yo dije 'iotra vez!', dije 'iAy no!, otra vez, ya no, pero pues ya era más que, este, pues ya, ya sabía, casi casi ya estaba segura que padecía de diabetes, porque sí sabía que empecé mi embarazo con sobrepeso, dije 'es lo más seguro', y que cuando nació mi hija me dijeron que si me volvía a embarazar se volvería a presentar, lo más probable era que se presentara de nuevo la diabetes gestacional, entonces ya estaba yo casi segura de que, de que iba a ser otra vez lo mismo. (31 SDG)

Como podemos ver, la presencia de la DMG provocó en ellas sentimientos de miedo y preocupación que las lleva a cuidar sus hábitos de alimentación, haciendo conciencia del tipo de comida que consumen. En otro sentido, las mujeres que tuvieron antecedentes de DMG, estuvieron conscientes de las posibilidades de volver a presentar la enfermedad en embarazos subsecuentes, de la importancia de los cuidados, y ante la falta de estos cuidados, las posibles consecuencias tanto para ellas como para sus hijos.

\section{Percepción de los riesgos}

por la presencia de la DMG

En el caso de las mujeres participantes, todas ellas tenían cierta idea de las complicaciones asociadas con la DMG tanto para ellas como para su hijo. Entre las más nombradas se encontraron: 1) tener un hijo con sobrepeso, y 2) que la madre sea propensa a desarrollar DM2 en un futuro. Algunas de ellas consideraron como otro riesgo la presencia de la DMG en embarazos futuros.

Es interesante que para las mujeres la preocupación, y por ende los riesgos de la enfermedad se enfocaron en las repercusiones principalmente del hijo. Tenían presente la posibilidad de que sus hijos al nacer tuvieran que quedarse internados para ser monitoreados por el personal médico; o el riesgo de que el bebé en un futuro desarrollara diabetes; asimismo, todas ellas co- 
incidieron que en los casos más graves podría fallecer el bebé:

Pues puede tener, o sea, el bebé primero es de, pues crece demasiado a la hora de nacer, a veces llegan a pesar más de 4 kilos, y con el tiempo la posibilidad de que ellos tengan la diabetes aumenta, es más posible que ellos llegan a tenerla, o incluso en el dado de los casos llegan a morir porque pues se esfuerzan demasiado, están muy grandes, es de, pues es otra amenaza que pues es el peor de los riesgos de tener diabetes gestacional, que muere el bebé. Y uno se ve muy grande, y puede dar es de, hasta en dado caso creo que la preecamplsia, la verdad no sé qué es eso pero, dicen que igual se hinchan. (Raquel, 39 SDG)

Ante dichos riesgos, las mujeres están convencidas de la importancia de la dieta que tiene que llevar como parte del tratamiento:

Entonces si no sigo la dieta, puede pasar lo que dijo el doctor. Por ejemplo, que nazca con retraso, o que nazca muerto. (Marta, 34 SDG)

\section{Conductas de autocuidado: cambios \\ en la alimentación y en la actividad física}

La dietoterapia es la primera etapa del tratamiento de la DMG, y representa el componente más importante. Es recomendable restringir el consumo de hidratos de carbono y dependiendo del peso de la mujer, a veces se recomienda también una restricción energética. En general, el tratamiento de estas mujeres incluye recomendaciones de una dieta correcta, promoviendo el consumo de grasas saludables e hidratos de carbono saludables (alimentos con alto contenido de fibra y bajo índice glucémico).

Las mujeres nos relataron qué tipo de alimentos incluyeron en su dieta para cumplir con el plan de alimentación recomendado, siendo las frutas y verduras parte de éste. En general los alimentos que aumentaron fueron las verduras, frutas y cereales integrales. De las verduras que comían con más frecuencia mencionan las siguientes: chayote, brócoli, calabaza, espinacas, ejotes, lechuga, coliflor y acelgas:

Bueno, sífueron verduras y frutas, porque casi yo de frutas no era mucho. Pero ahorita, sí aprendi a comer más fruta y pues verdura y más es de integral. (Clara, 35 SDG)

[...] estoy incrementando más la verdura, en lugar de hacer sopa de pasta, en la semana una vez sopa de pasta y todos los demás, hago sopa con chayotes y acelgas, o sopa con calabacita, o sopa de verduras, o sopa de coliflor con zanahoria, o de lechuga. (Marta, 34 SDG)
En el régimen de alimentación, no se permite que las mujeres consuman productos con alta cantidad de azúcar, y muy alto índice glucémico o grasa, por lo que tenían que disminuir algunos alimentos o dejarlos de consumir. Los ejemplos más comunes que refirieron dentro de los alimentos restringidos fueron: zanahoria, papas fritas, chocolate, tamales, papas, agua de sabor, quesadillas, camotes, betabeles, tacos, tortas, comidas capeadas, empanizadas, comidas grasosas, pan dulce, helados, pasteles, queso gouda, queso amarillo, galletas, el refresco y dulces. Para la mayoría de las mujeres, se les hacía difícil dejar de comer estos alimentos, así que, como una alternativa, solo disminuían el consumo de éstos.

Ahora bien, es interesante que a veces la disminución en el consumo de estos alimentos no se atribuyera por completo a la presencia de las DMG, sino que también se asoció con la presencia de síntomas al embarazo (náusea, vómito) es decir, no se les antojaba:

Pues más que nada el pan y el refresco y la grasa $y$ todo eso de la calle pero porque ahorita no se me antoja o sea para nada, la verdad me da mucho asco, mucho asco, los tacos de aquí a la vuelta, las quesadillas las veo y me da muchísimo asco, entonces yo digo que es por el embarazo porque a lo mejor si estuviera normal sí se me antojaría. (Clara, 35 SDG)

Si reflexionamos sobre la anterior narrativa, podemos ver que la "normalidad" a la que hace referencia la informante (no estar embarazada) es la que la lleva a disminuir el consumo de alimentos y no la enfermedad como tal, en ese sentido también la percepción de evitar consumir ciertos alimentos en mujeres con DMG no necesariamente se determina por un interés hacia la salud, sino a los síntomas de su estado reproductivo.

Sin embargo, también se observaron intentos de eliminar aquellos alimentos que no debían consumir:

[...] el refresco definitivamente sí lo he dejado; el refresco a lo mejor en la semana eran como 3 ó 2 $y$ ahorita sí definitivamente para nada, o de repente cuando vamos a comer a la calle o así de una fiesta o algo, si trato de pedir el refresco light y ni siquiera me lo acabo todo nada más le doy unos traguitos y ya para que se me quitara la sensación. (Clara, 35 SDG)

Ahora bien, se tiene que considerar que las conductas de autocuidado también están permeadas por los espacios sociales en lo que se desenvuelven las mujeres. Por ejemplo, una de ellas al trabajar en una tienda de abarrotes, menciona 
que a pesar de haber disminuido el consumo de tortillas, papa fritas, refrescos, y dulces, le costó mucho trabajo hacerlo, pues en su espacio laboral tenía al alcance todos esos productos:

Ay, pues ni modo, y es más difícil, se me hace mucho más difícil estar todo el día en la tienda, entonces, que veo las galletas, que el refresco, que las papas, que iay! no, se me hace muy pesado, tengo que poder. (Eleonor, $31 \mathrm{SDG}$ )

Los alimentos tradicionalmente consumibles fueron de los más difíciles de eliminar:

[...] la tortilla era lo que, es lo que me ha costado mucho trabajo dejar, la tortilla y el picante, pues este, es lo único que me ha costado mucho trabajo dejar...nada más tengo permitido una, si me como una tortilla ya no puedo comer galleta, ya no puedo comer una rebanada de pan, ni nada, entonces este es lo que me ha costado trabajo pero ya, ya casi me acostumbro, si ya casi. (Eleonora, 31 SDG)

En general podemos observar que en su mayoría las mujeres perciben que el objetivo no es renunciar completamente a comer ciertos alimentos, sino, disminuir su consumo. Como dice Silvia:

He procurado seguir mi dieta como me la mandaron pero luego si se me antoja mucho que una galleta nada más le doy una probadita... la zanahoria me gusta mucho, pero pues ahorita no la puedo comer...ya no me puedo comer que unas papitas, que un chocolatito que un tamalito, que esto, mi cafecito tampoco. (30 SDG)

Otra de las conductas que llevaron a cabo para "cumplir" con su dieta fue el cambio en la preparación de la comida y en el tamaño de las porciones:

[...] no hago capeados, ni exceso de grasa porque sí podemos comer grasa, pero una cucharita para que no se peguen los alimentos y todo, y es de, y pan en exceso, pues sí puedo comer uno, la mitad de un bolillo, pero quitarle toda la masa, la mitad, es de, en sí, puede probar uno de todo, pero no comerlo en exceso ni en abundancia... el pan, o sea, si voy a comerme un pedacito de pan de dulce, pues, menos de una cuarta parte, de uno. Es de, pero ya no me voy a comer ni el pan integral, ni galletas, una sola cosa. Es de, prácticamente todo, menos papa, camote, betabel, es lo que no podemos comer. (Raquel, 39 SDG)

Además de una adecuada selección de alimentos, y de disminuir la cantidad de algunos alimentos, las mujeres también reciben la recomendación de comer en horas definidas (tres comidas fuertes y dos colaciones) para distribuir adecuadamente el consumo de hidratos de carbono a lo largo del día y evitar picos de glucemia. Esto se reportó como algo difícil de lograr sobre todo en aquellas mujeres que laboran fuera del hogar:

No, no, es que por ejemplo este $\mathrm{mmm}$ voy a dejar a mi hija a la escuela, este, que si hay junta pues o algo ya regreso como a las 10 y media 11, entonces este tengo que abrir la tienda, que le barro, que esto, entonces yo ya voy desayunando es casi comida a las 12, entonces este, y ya después me pongo a hacer mi comida, que voy por la niña a la escuela y como hasta que cierro de la tienda, hasta las 9. (Eleonora, $31 \mathrm{SDG}$ )

O se les complicaba llevar un horario cuando tenían cita para su control prenatal:

Por ejemplo ahorita que he estado viniendo bien seguido aquí al hospital, en esta semana ya llevo 3 veces, la otra semana que viene me toca venir 2 veces y la otra que viene me toca venir el 24, o sea, que no puedo llevar un ritmo de horarios y que sí me gustaría porque así ya te vas controlando (se refiere a su hambre). (Silvia, 30 SDG)

Como podemos observar, para este grupo de mujeres realizar cambios en su alimentación no ha sido fácil, lo cual se explica por la diversidad de factores respecto a las costumbres que afectan la selección adecuada de tipo y cantidad de alimentos, la preparación de alimentos y los horarios definidos.

Como parte de las prácticas de autocuidado, la actividad física es un componente esencial. La Asociación Americana de Diabetes recomienda que las mujeres con DMG realicen ejercicio moderado siempre y cuando no esté contraindicado por el médico. En este estudio, se encontró que dos mujeres realizaban caminatas mientras tres mujeres no hacían ejercicio: dos por falta de tiempo, y una lo tenía prohibido por el médico:

Ay, es lo mismo (risas), lo mismo, no, bueno el tiempo que tengo según para ir a caminar porque tengo que caminar media hora, es cuando voy por la niña a la escuela, entonces voy caminando y me regreso caminando, pero así que dedique un tiempo a nada más ejercicio, ¡no! (Eleonora, 31 SDG)

Una de ellas comenta del por qué le prohibieron realizar ejercicio:

Uh huh, porque depende, porque como le dije que tenía cerclaje, porque la otra vez el doctor me dijo, 'bueno, haga su dieta y haga un poco, después camine,' le digo, 'es que me hicieron cerclaje,' 'No, entonces, no camines.'.. Y es de, y ahora me dijeron, 'no es de, caminatas, no subir ni bajar escaleras, no, o sea cuidados extremos.' Entonces pues yo creo que también ha funcionado porque ya llegué a la, a tantos meses que nunca había llegado. (Raquel, 39SDG)

Silvia caminaba 30 minutos porque era todo lo que podía aguantar. 
Pues procuro hacer lo que me dice mi doctor, salgo a caminar unos 30 minutos, bueno desde que yo me enteré que tenía la esta diabetes gestacional... me hubiera gustado saber qué otros ejercicios podía haber hecho, pero no tuve la oportunidad... (30 SDG)

Igual que Silvia, Martha reportó que caminaba los cincos días de la semana:

Unos treinta minutos... allí en el trabajo me pongo a caminar un rato o si no me da tiempo en el trabajo, allí en la casa me pongo a caminar y ya después de hacer mis cosas, me pongo a caminar un rato allí en la casa. (34 SDG)

Como se puede observar el ejercicio físico ciertamente sí estuvo presente, algunas realizaron la actividad en mayor tiempo que otras; $y$ solamente una de ellas no realizaban dicha actividad por restricción médica.

\section{Discusión}

Los resultados de este estudio cualitativo muestran que el conjunto de percepciones de las mujeres respecto a la presencia de la DMG, de los factores de riesgos y las posibles consecuencias de la enfermedad, las llevan a considerar a la alimentación y la actividad física fundamentales en la mejora del control de su enfermedad. Sin embargo dichas percepciones no necesariamente parecen traducirse en adecuada adherencia al tratamiento. El cumplimiento de las recomendaciones dietéticas en su mayoría fue referido como parcial. Aunque se observó que las mujeres sí están realizando cambios graduales y parece que se están moviendo hacia la conducta buscada. En la literatura esto se conoce como etapas de cambio y es parte del modelo transteórico del cambio de conducta ${ }^{22}$. El paciente siempre estará en algún lugar entre la negación a cambiar y el establecimiento del cambio y se sabe que para lograr cambios permanentes en el estilo de vida, estos deben de ser graduales ${ }^{23}$. El logro de un cambio pequeño puede aumentar la autoeficacia en el paciente y motivarlo a continuar con otro pequeño cambio, haciendo del cambio de conducta un proceso, que poco a poco vaya promoviendo un cambio de mayor impacto y que sea mantenido a largo plazo $^{24}$

Considerando el modelo de atención en nuestro país, en el cual el enfoque es primordialmente curativo y general, en vez de preventivo, específico e individual, es importante generar nuevas estrategias en las cuales se considere al tratamiento como un proceso de cambio. Una de las estrate- gias más utilizadas para lograr modificaciones en la alimentación y que es parte de algunas teorías del cambio de conducta, es el establecimiento de metas a corto plazo, realistas y sencillas ${ }^{25}$. Las mujeres entrevistadas en este estudio refirieron un grado de cumplimiento muy diferente ante las distintas recomendaciones dietéticas y de actividad física.

Es bien sabido que la adherencia al tratamiento en pacientes con DM varía mucho entre los diferentes componentes del tratamiento. La adherencia a una recomendación no necesariamente predice la adherencia a otra. Tanbien se sabe que el lograr una adherencia a la dieta es complicado, debido a varios factores externos que afectan el cambio de conducta. En este grupo de mujeres con DMG, se observó que aunque no dejan de consumir los alimentos "prohibidos" ellas hacen un esfuerzo para cumplir con el tratamiento, mediante la disminución del consumo de los mismos (cantidades) y/o modificando las preparaciones de sus alimentos. Lo anterior, ciertamente está relacionado, entre otras cosas a la tradición alimentaria la cual tiene su referente en la historia política, vicisitudes culturales, económicas e instituciones de cada sociedad. Es decir, el consumo de los alimentos es una serie de condicionamientos culturales que determinan la exclusión o preferencia de los alimentos - relacionado con el gusto o antojo - según el significado de los rituales sociales, el mensaje que se intercambia al compartir los alimentos, así como los valores éticos e incluso religiosos ${ }^{26} \mathrm{y}$ en ocasiones por su condición reproductiva. Además el nivel socioeconómico determina la selección de alimentos. Estos aspectos deben ser considerados por el profesional de la salud que establece las recomendaciones de alimentación, ya que muchas veces el prohibir alimentos no soluciona la problemática, mientras que la inclusión de otras estrategias, que tomen en cuenta la situación individual (familiar, social, cultural y económica) de las mujeres, puede ayudar a favorecer cambios positivos más graduales en la alimentación.

En ese mismo sentido, las percepciones sobre evitar el consumo de ciertos alimentos ya sea por la presencia de los síntomas del propio embarazo (no antojos de ciertos productos) o por la DM en sí, es de considerarse, pues invita a que los profesionales de la salud indaguen a profundidad los motivos del consumo o no consumo de alimentos, para considerarlos al establecer las estrategias de tratamiento. Se recomienda que la adherencia se evalúe por conductas específicas, y por lo tanto que el tratamiento médico y nutricio 
también se dirija a la modificación de una o dos conductas importantes ${ }^{23}$.

En este estudio se exploraron en profundidad las percepciones de las mujeres en cuanto a al auto-manejo de su enfermedad, y se corroboró lo que se ha descrito en la literatura sobre los múltiples factores que afectan el que una mujer sí lleve a cabo las recomendaciones de alimentación y actividad física ${ }^{24,27,28}$. En un estudio realizado en mujeres con DMG dentro del mismo hospital, se establece la necesidad de tomar en cuenta los factores psicosociales, las barreras, los motivadores, y la autoeficacia al evaluar la adherencia al tratamiento médico y nutricio ${ }^{29}$.

En este grupo de mujeres entrevistadas con DMG se observó que parecen estar conscientes de algunos factores que provocan la enfermedad (herencia, sobrepeso u obesidad, prácticas de alimentación).

Las emociones y los sentimientos, que son parte de la estructura de los sujetos ${ }^{30}$, y representaron un papel fundamental en la conducta de autocuidado de las mujeres con DMG, pues las ayudaron a comprender la importancia de los tipos de alimentos y las cantidades que deben ingerir. Las emociones fueron factor clave para que llevaran a cabo la dieta. Los miedos y preocupaciones respecto a los riesgos de la DMG sobre ellas, y en particular en sus bebés, las llevó a hacer un esfuerzo por tratar de cumplir al "pie de la letra" con las recomendaciones dietéticas. En este sentido, la educación médica y educación en nutrición, deberían ejercerse dentro de un contexto de confianza, que sea sensible a su contexto social y cultural, y que empodere a las mujeres y no que las asuste. También se percibió frustración sobre todo al tratar de eliminar los alimentos tradicionalmente consumibles ${ }^{12,13}$.

Las percepciones y vivencias de tener DMG, al menos para este grupo, muestra la complejidad del autocuidado (adherencia a la dieta y realización de ejercicio), el cual requiere de cambios de conducta. El tratamiento médico y nutricio de la DMG debe incluir estrategias de intervención que incluya componentes conductuales para facilitar el cambio (establecimiento de metas, automonitoreo, entre otros). Un programa de terapia médica y nutricia para embarazadas con $\mathrm{DM}$ (DMG y DM2), implementado en el Instituto Nacional de Perinatología, disminuyó de manera importante algunas complicaciones perinatales (preeclampsia, hospitalización materna por descontrol glucémico y muerte neonatal). Los objetivos de este programa fueron promover una ganancia de peso adecuada y un control glucémico óptimo y la consulta de nutrición fue frecuente (cada 2 o 3 semanas) e incluyó el establecimiento de metas individuales, así como un componente educativo dirigido a aumentar conocimientos y desarrollar habilidades para lograr el automanejo ${ }^{31}$. Es necesario diseñar e implementar programas dirigidos a mujeres embarazadas con DM, que incluyan estrategias para individualizar las recomendaciones de alimentación y actividad física tomando en cuenta el contexto sociocultural de las mujeres, así como sus preferencias y hábitos previos. El lograr cambios positivos en la alimentación se refleja en un control metabólico óptimo, el cual puede ayudar a romper el efecto intergeneracional de la enfermedad.

\section{Colaboradores}

M Chávez-Courtois, C Grahan, I Romero-Pérez, G Sánchez-Miranda, B Sánchez-Jiménez y O Perichart-Perera han participado igualmente en cada una de las etapas de elaboración de este artículo. 


\section{Referencias}

1. American Diabetes Association (ADA). Gestational Diabetes Mellitus. Diabetes Care 2004; 27(Supl. 1):S88-S90.

2. Metzger B, Buchanan TA, Coustan DR, de Leiva A, Dunger DB, Hadden DR, Hod M, Kitzmiller JL, Kjos SL, Oats JN, Pettitt DJ, Sacks DA, Zoupas C. Summary and Recommendations of the Fifth International Workshop-Conference on Gestational Diabetes Mellitus. Diabetes Care 2007; 30(Supl. 2):S251-S260.

3. Kjos S, Peters RK, Xiang A, Henry OA, Montoro M, Buchanan TA. Predicting future diabetes in Latino women with gestational diabetes: utility of early postpartum glucose tolerance testing. Diabetes 1995 44(5):586-591.

4. Gillman M, Rifas-Shiman S, Berkey CS, Field AE, Colditz GA. Maternal gestational diabetes, birth weight, and adolescent obesity. Pediatrics 2003; 111(3):e221-226.

5. Jimenez-Cruz A, Bacardi-Gascon M. The fattening burden of type 2 diabetes on Mexicans. Diabetes Care 2004; 27(5):1213-1215.

6. Olaiz G, Rivera J, Shamah T, Rojas R, Villalpando S, Hernández M, Sepúlveda J, editores. Encuesta Nacional de Salud y Nutrición 2006. México: INSP; 2006.

7. Fausto J, López RMV, Rodríguez MGA, Zermeño MCL. Antecedentes históricos y sociales de la obesidad en México. Investigación en Salud [artículo en Internet]. 2006 Ago [acceso 2011 Dic 12]. Disponible en: http://www.medigraphic.com/pdfs/invsal/ isg-2006/isg062f.pdf

8. Huidobro A, Fulford A, Carrasco E. Incidencia de diabetes gestacional y su relación con la obesidad en embarazadas chilenas. Rev Méd Chile 2004; 132(8):931-938.

9. Jones E, Roche C, Appel S. A Review of the Health Beliefs and Lifestyle Behaviors of Women with Previous Gestational Diabetes. JOGNN 2009; 38(5):516526.

10. Hjelm K, Nyberg P, Isacsson A, Apelqvist J. Beliefs about health and illness essential for self-care practice: a comparison of migrant Yugoslavian and Swedish diabetic females. JAN 1999; 30(5):1147-1159.

11. Hjelm K, Bard K, Nyberg P, Apelqvist J. Religious and cultural distance in beliefs about health and illness in diabetic women of different origin living in Sweden. IJNS 2003; 40(6):627-643

12. Daniulaityte R. Making sense of diabetes: cultural models, gender and individual adjustment to type 2 diabetes in a Mexican community. Soc Sci \& Medicine 2004; 59(9):1899-1912.

13. Carbone E, Rosal MC, Torres MI, Goins KV, Bermudez OI. Diabetes self-management: perspectives of Latino patients and their health care providers. $\mathrm{Pa}$ tient Education and Counseling 2007; 66(2):202-210.

14. Kieffer E, Willis SK, Arellano N, Guzman R. Perspectives of pregnant and postpartum Latino women on diabetes, physical activity, and health. $H E B$ 2002; 29(5):542-556

15. Kieffer E, Sinco B, Kim C. Health behaviors among women of reproductive age with and without history of gestational diabetes mellitus. Diabetes Care 2006; 29(8):1788-1793
16. Pérez S, Vega-García L, Romero-Juárez G. Prácticas alimentarias de mujeres rurales: ¿una nueva percepción del cuerpo? SPM 2007; 49(1):50-62.

17. Horton E. Exercise in the treatment of NIDDM. Applications for GDM? Diabetes 1991; 40(2):S175-S178.

18. Jovanovic-Peterson L, Peterson C. Review of gestational diabetes mellitus and low-calorie diet and physical exercise as therapy. Diabetes Metabol Rev 1996; 12(4):287-308.

19. Instituto Nacional de Perinatología (INPer). Аnиario Estadístico 2008. México: INPer; 2008.

20. Ramírez M. Diabetes mellitus gestacional. Experiencia en una institución de tercer nivel de atención. en Ginecol Obstet Méx 2005; 73(9):484-491.

21. Taylor SJ, Bogdan R. Introducción a los métodos cualitativos de investigación. La búsqueda de significados. Barcelona: Paidós; 1992

22. Prochaska J, DiClemente C. The transtheoretical approach. In: Norcross J, Goldfried M, editors. Handbook of psychotherapy integration. New York: Oxford University Press; 2005. p. 147-171.

23. Koenisberg R, Bartlett D, Cramer J. Facilitating treatment adherence with lifestyle changes in diabetes. AFP 2004; 69(2):309-316.

24. Hill J. Can a small-changes approach help address the obesity epidemic? A report of the Joint Task Force of the American Society for Nutrition, Institute of Food Technologists, and International Food Information Council. AJCN 2009; 89(2):477-484.

25. Shilts M, Horowitz M, Townsend M. Goal Setting as a Strategy for Dietary and Physical Activity Behavior Change: A Review of the Literature. AJHP 2004. 19(2):81-93.

26. Montanari M. Historia, alimentación, historia de la alimentación. Sánchez J, coordinador. Problemas actuales de la historia Nistal. Salamanca: Universidad del Salamanca; 1993. p. 19-28.

27. Tillotson L, Smith M. Locus of control, social support, and adherence to the diabetes regimen. TDE 1996; 22(2):133-139.

28. Wooldridge K, Wallston KA, Graber AL, Brown AW, Davidson P. The relationship between health beliefs, adherence, and metabolic control of diabetes. TDE 1992; 18(6):495-500.

29. Balas-Nakash M, Rodríguez-Cano A, Muñoz-Manrique $C$, Vásquez-Peña $\mathrm{P}$, Perichart-Perera $\mathrm{O}$. Tres métodos para medir la adherencia a un programa de terapia médica y nutricia en mujeres embarazadas con diabetes y su asociación con el control glucémico. Rev Invest Clin 2010; 62(3):235-243.

30. Le Breton D. Las pasiones ordinarias. Antropología de las emociones. Buenos Aires: Nueva Visión; 1998.

31. Perichart-Perera O, Balas-Nakash M, Parra-Covarrubias A, Rodriguez-Cano A, Ramirez-Torres A, Ortega-González C, Vadillo-Ortega F. A medical nutrition therapy program improves perinatal outcomes in Mexican pregnant women with gestational diabetes and type 2 diabetes mellitus. TDE 2009; 35(6):1004-1013.

Artigo apresentado em 27/03/2013

Aprovado em 25/05/2013

Versão final apresentada em 04/06/2013 